\title{
Significacion didactica y de impacto social de la conformacion del Comité Tecnico de Emergencias UMSA, durante la crisis del agua 2016 de La Paz, Bolivia
}

Maria Nadiezda Otero Valle ${ }^{1}$

https://orcid.org/0000-0002-3078-5115

\section{Resumen}

La era actual del Antropógeno viene con la alteración de los sistemas hídricos por contaminación y variación en su disponibilidad, que exige la actualización e innovación de la formación de la ingeniería hidrosanitaria en relación con la gestión integral del agua incluyendo la gestión de riesgos, ambiental y resiliencia al cambio climático. La investigación cualitativa sobre la inserción de la gestión integral del agua en la educacion de la ingeniería hidrosanitaria ha ido identificando, a partir del 2009, aspectos que han contribuido a la propuesta de conformación del Comité Técnico de Emergencias UMSA en la Crisis del Agua del 2016, y es caso de estudio del presente artículo, con enfoque interpretativo; donde se moviliza la comunidad de la Universidad Mayor de San Andres UMSA, con el objetivo de contribuir, en el menor tiempo posible, a la solucion de la peor crisis por falta de agua de los últimos 25 años que ponía en riesgo la salud de la población (300.000 habitantes aprox.) de la zona sur de La Paz . El trabajo de esta comunidad en acción se constituye en una alternativa integral de interacción social, enseńanza-aprendizaje, investigacion de impacto social.

Palabras claves: Didáctica, Gestión integral del agua, Comunidad en accion.

\section{Didactic and social impact significance of the UMSA Technical Emergency Committee, during 2016 water crisis in La Paz, Bolivia}

\section{Abstract}

The Anthropogenic current era comes with the alteration of water systems by pollution and variation in their availability, which requires the updating and innovation of the hydrosanitary engineering education in relation to integrated water management including risk management, environmental and resilience to climate change. Since 2009 the qualitative research on the integrated water management insertion into the education of hydrosanitary engineering, has been identifying aspects that have contributed to form the UMSA Technical Emergency Committee during 2016 Water Crisis to which this article refers, us a case study with an

\footnotetext{
${ }^{1}$ Docente Emérito Facultad de Tecnologia Universidad Mayor de San Andres UMSA, Estudiante del Programa Doctorado no Escolarizado en Educación Superior del Centro Psicopedagógico y de Investigación en Educación Superior (CEPIES/UMSA), Msc in Hydrology (VUB-Brussels), Ingeniero civil UMS; Email: mnotero13@gmail.com.
} 
interpretative approach; where the community of the Universidad Mayor de San Andres UMSA is mobilized, for contributing to the emerging crisis solution, in the shortest possible time, when the southern area of La Paz population (300,000 inhabitants approx.) are in health risk due to lack of water. The work of this community in action is an integrated alternative to social interaction, teaching and research with impact on population.

Keywords: Didactic, Integrated water management, Community in action.

\section{Introduccion}

Las actividades humanas han roto las dinámicas de los sistemas y ecosistemas. Además, que el desarrollo tecnológico ha ido provocando cambios acelerados, culturales, desestabilizando costumbres, normas culturales. La reacción a esta complejidad no es la adaptación, sin embargo, la educación puede dar las herramientas necesarias para ello a pesar de que la ingeniería y los sistemas tecnológicos están fuera de contexto cultural, social e institucional será necesario iniciar la provisión de marcos teóricos para entender, comunicar y solucionar problemas complejos con significancia y dimensión tecnológica. (Allenby, 2011)

La investigación de la inserción de la gestión integral del agua en la ingeniería hidrosanitaria, que se realiza en CEPIES-UMSA, surge como preocupación de actualizar y renovar la educación de la ingeniería hidrosanitaria UMSA en relación con los nuevos retos que conllevan las actuales alteraciones de los sistemas hídricos, el avance de la ciencia-tecnologia y los contextos cultural, social e institucional. La gestión integral del agua y su educacion tienen una perspectiva de complejidad.

En especial, en el tema de los sistemas hídricos es vital lograr la transformación educativa, que acompañe los "cambios acelerados de la sociedad, tecnologia“ (Allenby, 2011) y ecosistemas para poder contribuir a la disminución del deterioro ambiental, efectos por cambio climático y desastres correspondientes.

La formación de la ingeniería sanitaria y ambiental dentro la Universidad Mayor de San Andres ( UMSA) mantiene sus objetivos iniciales desde la década 70: "estudiar, proyectar, construir, operar y mantener todas aquellas obras programas de saneamiento ambiental, en especial aquellos relacionados con el abastecimiento de agua potable y los sistemas de alcantarillado y eliminación de excretas“ (Rivas Mijares, 1966, pág. 4), con implementaciones posteriores hacia la formación social del ingeniero hidrosanitario en proyectos rurales de participación comunitaria de las 
décadas 80 y 90; y relacionadas algunas a las agendas de desarrollo sostenible de la década 2000.

El quehacer ingenieril implica posicionarse técnica, política e ideológicamente en relación con temas que emergen actualmente dentro de las profesiones vinculadas a la ciencia y la tecnología. Asimismo, significa imaginar /apostar a un perfil de ingeniero que pueda tomar decisiones técnicas situadas con un pensamiento crítico, que realice una lectura de la realidad que lo interpele para constituirse en sujeto que opte por actuar por el bien común (Stella Maris Abate \& Cecilia Veronica Lucino, 2017).

El concepto de gestión integral del agua ha sido abordado desde una perspectiva de complejidad que reintegre la naturaleza del patrimonio hídrico y sus cambios en el tiempo por las continuas alteraciones de los ecosistemas por las actividades socio-economicas (Vasquez Paniagua , 2017) y en esa dimensión la educación podría transformarse. Ver figura 1.

Parte de la investigación es identificar, interpretar y evaluar, de forma sistemática (Atlas.ti) los escenarios didácticos: de enseñanza-aprendizaje, interacción social, investigación, de la educación de la ingeniería hidrosanitaria incluyendo los espacios laborales donde se continua la formacion.

La interacción social en la UMSA es una de las actividades fundamentales de la vida universitaria junto a la formacion de profesionales comprometidos y la investigación orientada a solucionar los problemas, necesidades y demandas del pueblo boliviano (Paz Rada, 2012).

La formacion dentro la interacción social y durante el servicio profesional se realizan entorno a comunidades de aprendizaje, que se constituyen en

La comunidad de práctica en el contexto de la educación superior es un grupo de personas (docentes e investigadores) unidas por intereses comunes para compartir y construir de forma colaborativa conocimientos especializados, intercambiar información y experiencias sobre la propia práctica profesional, interactuar para seguir aprendiendo y relacionarse entre sí, de tal manera que se desarrolla un repertorio común de pensamiento y acción y se constituyen así espacios para la mejora de la formación docente y el desarrollo profesional (Bozu \& Ibernon Muñoz, 2009)

En el presente caso se denominarán "comunidades en accion".

El presente artículo se refiere al caso de estudio: Conformación del Comité Técnico de Emergencias en la Crisis del Agua del 2016 en la UMSA en la peor crisis por falta de agua de los últimos 25 ańos. 
(Otero Valle, Propuesta de conformacion del Comite Tecnico de Emergencias en la Crisis del Agua, 2018).

Figure 1. Red semantica del Concepto tiempo y Gestion Integral del Agua. Elaboracion Propia. Fuente. (Vasquez Paniagua , 2017)

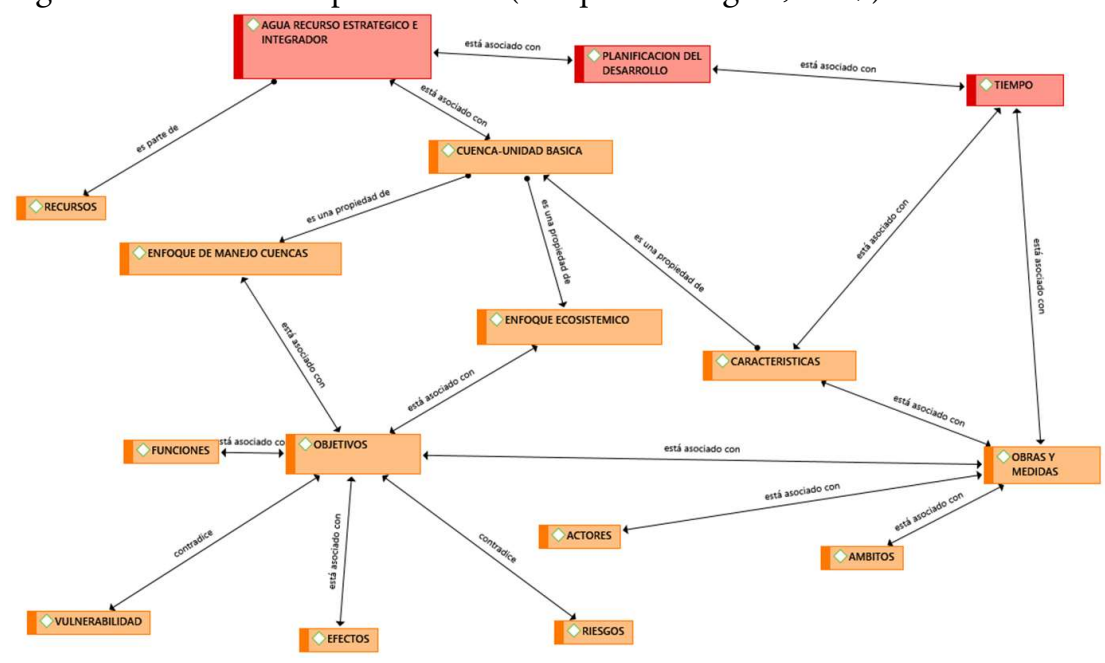

\section{Objetivo}

El propósito del presente artículo es describir, interpretar y analizar la significancia didáctica y de impacto social de la conformación del Comité Técnico de Emergencias en la Crisis del Agua del 2016 en la UMSA, donde se conforma una "comunidad en acción emergente" como una alternativa integral de enseñanza-aprendizaje, interacción social e investigación aplicada en la formación de la ingeniería hidrosanitaria sostenible

El comité realizo un trabajo participativo centrada en las actividades investigacion e interacción social para poner en práctica su experiencia. Con estructuras de participación multiple centrada con una organización definida en base a la producción de conocimiento de la problemática del agua.

Los mecanismos de reproducción y crecimiento se establecen mediante la interacción, intercambio de conocimientos y practicas continuas sobre la problemática del agua 


\section{Estrategia emergente y metodologia}

El estudio de caso se realiza dentro la investigación cualitativa, con enfoque interpretativo busca la identificación e interpretación del significado de la didáctica integral y el impacto social, que se manifiesta durante el desarrollo de las actividades del Comité Tecnico de Emergencias UMSA en una situación crítica. El investigador es observador participante.

Los sujetos del estudio de caso son docentes especializados, autoridades académicas y científicas de los institutos de investigación que interactúan en forma directa o mediante la prensa con los niveles de decisión del sector agua y sus acciones se amplían hacia la comunidad universitaria y la sociedad relacionada a la temática y problemática del agua

\section{¿Qué características tuvo el Comité Tecnico de Emergencias del Agua para constituirse en ¿̨una comunidad en acción para la gestión integral del agua?}

El comité realizo un trabajo participativo centrada en las actividades investigacion e interacción social para poner en práctica su experiencia. Con estructuras de participación multiple centrada con una organización definida en base a la producción de conocimiento de la problemática del agua.

Los mecanismos de reproducción y crecimiento se establecen mediante la interacción, intercambio de conocimientos y practicas continuas sobre la problemática del agua. 
Figure 2. Características del Comité técnico de Emergencias del Agua. elaboración propia.

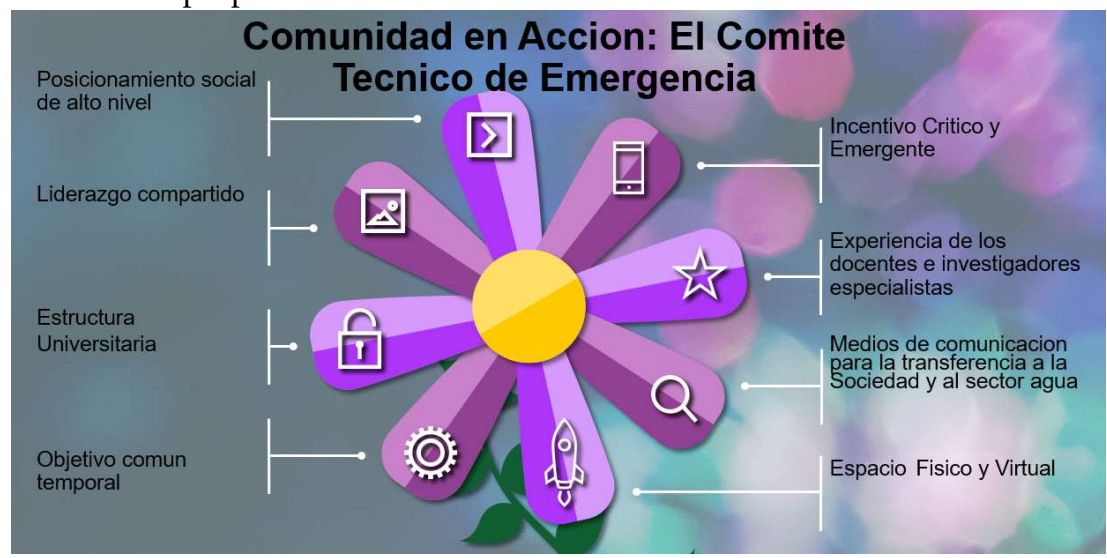

La conformación del Comité parte del objetivo de bien común : aportar a la solución del problema de corte del servicio de agua en 98 barrios de la zona sur de la Ciudad de La Paz, ante una población afligida por la falta de respuesta oportuna de las instituciones del sector.

La propuesta, de conformación del Comité se ha constituido en una estrategia emergente para asegurar la influencia de la comunidad academica y científica sobre las decisiones oportunas de los responsables de la operación del servicio y la atención de la emergencia, que no habían informado hasta ese momento a la población de solución alguna.

La comunidad academica, profesional y científica hasta antes de conformar el comité se había manifestado en los medios de comunicación, con ideas y propuestas de solución sin encontrar la atención correspondiente de las instituciones operativas del sector agua. Por lo que se había deducido la necesidad de conformar una propuesta de la UMSA al problema.

La propuesta, se basó en resultados logrados en la investigación de inserción de la gestión integral del agua en la educación de la ingenieria hidrosanitaria hasta el 2016 correspondientes a: Paz,

a) la contextualizacion de gestión integral del agua de la ciudad de La

b) el contexto enseñanza aprendiza, interacción social e investigación de la ingenieria hidrosanitaria de la UMSA

c) el desempeño de las instituciones del sector agua, 
d) los conocimientos de la gestión en riesgos en saneamiento básico del sector y EPSAS

e) identificación de necesidades de investigación y desarrollo en las temáticas de deterioro ambiental, cambio climático y crecimiento poblacional dentro la ingeniería hidrosanitaria sostenible.

El nivel de alta confianza de la sociedad hacia la UMSA; considerada entre las 3 instituciones más confiables en los años 2011 y 2012, dentro una encuesta realizada por la fundación IPSOS (Apoyo Opinion y Mercado) sobre la calidad de vida en el municipio de La Paz. (http://www.aquicomunicacion.umsa.bo/sociedad//asset_publisher/t1Gi/content/las-instituciones-de-mayor-confianza-son-laumsa-la-iglesia-y-el-gobierno-municipal)

La propuesta presentada a la máxima Autoridad de la UMSA, viabilizo la reunión magna conformada por especialistas docentes del área hidrosanitaria, como autoridades de los institutos de investigación relacionados a la temática del agua dentro la UMSA.

\section{Resultados}

El comité logro influir en la solución del problema mediante recomendaciones para el sector agua en durante la atencion de emergencias y acciones futuras, elaboración de cartilla técnica y el monitoreo de 17 vertientes y tanques de dotación agua.

Las recomendaciones de Atención de Emergencias para EPSAS y el Sector Agua y Saneamiento ; fueron de carácter técnico, social y de salud, relacionado al suministro del agua de calidad, conformación de un Comité de Emergencias Interinstitucional dentro la Empresa de Aguas (EPSAS), cumplimiento de las Normas Esfera, la atención de emergencias centrada en las personas (Mesa ASH).

La elaboración de la Cartilla Técnica para la potabilización de agua en domicilios, y logro involucrar a la población damnificada en forma activa en la solución del problema de emergencia.

El Monitoreo de 17 vertientes y tanques de dotación agua que permitió la evaluación hidroquímica y los consejos de uso de estas alternativas de abastecimiento en tiempos de emergencia para evitar riesgos en la salud. 


\section{¿Cuáles fueron las experiencias y lecciones aprendidas en la conformación del Comité de Emergencias?}

La solución definitiva del problema de falta agua, implicaba diversas acciones a corto y mediano plazo, porque se había identificado falencias importantes:

- Falta de planificación hidrológica en relación con la disponibilidad, manejo de los embalses y la demanda del agua.

- Falta de planificación de prevención de emergencias.

- Inadecuada Gestión Integral del agua, en un nuevo escenario: crecimiento de la población acompañado con deterioro ambiental, cambio climático que afectan a los sistemas hídricos, necesidades de ampliar los sistemas de abastecimiento de agua con tecnologías actualizadas.

La Universidad Mayor de San Andres, no es parte del grupo tecnológico, científico de la planificación, seguimiento y control de los proyectos y medidas de saneamiento básico del sector agua y EPSAS de La Paz. Y no logra hasta la fecha ser parte de.

La UMSA, para atender demandas posteriores de la sociedad boliviana, convirtió el Comité de Tecnico de Emergencias en la Comisión de Alto Nivel Multidisciplinario, que atendió en subcomisiones análisis de los proyectos de las Represas del Bala y Chepete (2019), Deslizamiento Relleno Sanitario Alpacoma y selección del área de reubicación (2019).

La UMSA se constituye un referente de interacción social en situaciones críticas.

\section{¿Cómo se ha generado el aprendizaje, investigación e interacción social integral?}

El aprendizaje emergente ha sido de carácter interactivo, participativo y multidisciplinario bajo la temática del agua dentro la comunidad universitaria, y ha ampliado la interacción hacia las instituciones gubernamentales en mesas de discusión técnicas principalmente.

La Facultad de Humanidades y Ciencias de la Educación ha sistematizado la información científica generada durante la Crisis del Agua por la comunidad universitaria en su publicación: Crisis del Agua; El acceso a la información científica sobre los recursos hídricos y en agua en La Paz, como parte del proyecto Crisis del Agua del Instituto de Estudios Bolivianos. 
Los institutos de investigación han ampliado sus proyectos $y$ fortalecidos laboratorios hacia la temática del agua en las diferentes facultades.

Los docentes, investigadores y alumnos han sido integrados a campañas de investigación e interacción social durante y después de la conformación del comité.

En los procesos de enseñanza y aprendizaje, se incorporan mayores temas relacionados a la investigación e interacción social.

\section{Conclusiones}

El Comité Técnico de Emergencias en la Crisis del Agua del 2016, se ha constituido en una autoridad técnica para la solución emergente. Y es un resultado alcanzado en la búsqueda de la inserción de la gestión integral del agua en la formación de la ingeniería hidrosanitaria de la UMSA (2009-2019) que se constituye una alternativa integral de aprendizaje de "comunidad en accion", donde se elabora la solución en un escenario académico, científico y profesional, de forma colaborativa, compartida e interactiva.

En posteriores años al 2016, se cambio el Comité de emergencias del agua, por Comité Multidisciplinario de la UMSA donde fueron atendidas demandas de la sociedad e institucionales como los casos de estudio del Deslizamiento del Relleno Sanitario Alpacoma (La Paz) y la conformación de la subcomisión de evaluación del proyecto hidroeléctrico Bala - Chepete.

Los institutos fueron ampliando sus investigaciones en el tema del agua, sin embargo, requieren mayores recursos.

La influencia sobre el bienestar de la sociedad en la Crisis del 2016 y el fortalecimiento de investigaciones en la UMSA respecto a la gestión integral del agua, gestión de riesgos, resiliencia climática, etc. valida a esta comunidad de acción como una alternativa integral de enseñanza-aprendizaje, investigacion e interacción social.

\section{Discusion}

La propuesta de conformación del Comité de Emergencias de la UMSA ha identificado la falta de una planificación conjunta entre miembros de comunidad universitaria (UMSA), la sociedad en general con las respectivas autoridades municipales, gubernamentales; donde la planificación de la 
gestión integral del agua debía ser el objetivo común, considerando los nuevos retos del antropógeno.

En el caso de la Gestion Integral de los recursos hídricos se tiene los consejos de cuencas (Programa Agua, 2017), pero no van acompañados de estructuras académicas, científicas que son necesarios para contribuir a las decisiones necesarias.

Lo que significa que tanto en las comunidades universitarias como en las comunidades locales: la investigacion, la interacción social y la didáctica debe ser estructurada de forma de que se realice la gestión integral del agua para tomar decisiones sostenibles.

La comunidad en acción tiene un carácter temporal y se activa ante un objetivo de bien común, por lo que la conformación Comité Multidisciplinario, tiene un tiempo de vida mayor por tanto su permanencia, implicaría llevar las investigaciones aisladas de cada Facultad a un Centro de Investigación Multidisplinario del Agua en la UMSA, que contribuya a la planificación del Sector del Agua.

Los sujetos del presente caso de estudio tendrían que participar en la política del agua, para asegurar la influencia sobre los derechos de acceso al agua (Morin, 2011), sin embargo, no sucede ello.

\section{Referências}

Allenby, B. The Theory and Practice of Sustainable Engineering, 2011.

Bozu, Z., \& Ibernon Muñoz, F. Creando Comunidades de practica y conocimiento en la Universidad una experiencia de trabajo entre universidades de lengua catalana. Revista de Universidad y Sociedad del Conocimiento, 2009. 1-10.

Morin, E. La Via para el futuro de la Humanidad. Madrid: Paidos, 2011.

Otero Valle, N. Propuesta de conformacion del Comite Tecnico de Emergencias en la Crisis del Agua. En M. Otero Valle, M. Garcia, G. Rodrigo Lira, R. Cortez, E. Ricaldi Yarvi, \& A. Gutierrez, Crisisi del Agua: El acceso a la informacion cientifica sobre los recursos hidricos y el agua en La Paz (págs. 1327). La Paz: Universidad Mayor de San Andres, 2018. ISBN 978-99954-4946-9.

Paz Rada, E. Una reflexion sobre las relaciones sociedad-UMSA: La Interaccion Social, 2012. Scielo, 91-109. Obtenido de 
http://www.scielo.org.bo/scielo.php?script=sci_arttext\&pid=S004029152012000100007\&lng=es\&nrm=iso >. ISSN 2413-5720

Programa Agua, C. y. Cusco. Obtenido de https://www.gwp.org/globalassets/global/gwp-sam_files/noticias/gest-recurhidricos.pdf

Rivas Mijares, G. Problemas de la Educacion en Ingenieria Sanitaria. Boletin de la Oficina Sanitaria, 1966. 4.

Stella Maris Abate \& Cecilia Veronica Lucino. La dimension social de la ingenieria. En Ingenieria y saberes sociales (pág. 106). La Plata: Edulp, 2017.

Xuzel Ana Villavicencio Peralta. Aporte de las ingenierías en los ODS. 2019. Obtenido de https://ucsp.edu.pe/la-importancia-de-la-ingenieria-en-eldesarrollo-sostenible/

Recebido em: 13 set. 2020

Aceito em: 07 dez. 2020 